\title{
Poor uptake of hepatitis B immunization amongst hospital-based health care staff
}

\author{
A.D. Burden and P.J. Whorwell
}

Department of Medicine, University Hospital of South Manchester, Manchester M20 8LR, UK

Summary: The uptake of hepatitis $B$ vaccine was assessed amongst 100 medical and 100 nursing staff in a teaching hospital with a policy of recommending to those at risk that they should seek immunization from their general practitioners.

Sixteen per cent of nurses and $31 \%$ of doctors had completed a course of immunization with confirmation of seroconversion. An additional $9 \%$ and $18 \%$ respectively had been immunized without post-immunization serology. Ninety three per cent of nurses and $61 \%$ of doctors who had not been immunized would like to receive the vaccine. The commonest reasons for non-immunization amongst nurses were fear of vaccine and lack of advice, and amongst doctors, apathy and difficulty in obtaining the vaccine. Eighty seven per cent of medical staff and $57 \%$ of nurses had a history of needle stick injury.

The low rates of vaccine uptake in this study combined with the high incidence of needlestick injury calls for a reappraisal of present hepatitis B vaccination programmes in hospitals. In particular the abrogation of responsiblity for immunization to general practitioners is probably a major disincentive to potential vaccinees.

\section{Introduction}

Hepatitis B is a preventable disease that presents an occupational hazard to health care and laboratory personnel. It is not only potentially fatal but also carries a risk of chronic infection and possible transmission to patients. ${ }^{1}$ During the 5 years between 1980 and 1984, 364 cases of acute hepatitis B were reported amongst health service staff in England, Wales and Ireland representing $5.4 \%$ of all cases of the disease. ${ }^{2}$ This figure may considerably underestimate the problem by failing to take account of subclinical infection and undernotification. ${ }^{3}$

Since 1987, a recombinant yeast-derived hepatitis B vaccine has been available which is relatively inexpensive. In addition it should not be associated with the fear of transmission of other infectious agents which has prevented some health care workers from receiving the previously available plasma-derived vaccine. ${ }^{4}$

Few studies have examined immunization rates amongst health care staff in the United Kingdom. It has been estimated that $24 \%$ of physicians and $40 \%$ of health care staff have been vaccinated in the USA, ${ }^{5}$ and in the UK a recent report reveals that $48 \%$ of general practitioners have been immunized. ${ }^{6}$

Correspondence: P.J. Whorwell, F.R.C.P.

Accepted: 17 October 1990
This study was undertaken to assess the rate vaccination amongst hospital-based medical and nursing staff and consider ways of improving $\overline{\bar{O}}$ uptake.

\section{Methods}

One hundred hospital doctors and 100 clinicalo nurses were randomly selected from the 160 medical and dental staff and 1,537 nurses and 3 midwives employed at the University Hospital of South Manchester. During January and February 3 . 1990, each subject was interviewed and the following data obtained: hepatitis B vaccination status, $\frac{}{3}$ vaccine advice, attitudes towards hepatitis $B$ and its $O$ vaccination, ease of obtaining vaccine (visual analogue score) and history of previous needlestick $\underline{\underline{O}}$ injury.

\section{Results}

Two hundred subjects completed the questionnaire with no refusals. The medical staff group consisted $\stackrel{\odot}{\bar{D}}$ of 25 consultants, 14 senior registrars, 14 registrars, $\stackrel{\odot}{\oplus}$ 28 senior house officers and 19 house officers representing all major clinical specialties. Amongst ${ }^{\circ}$ the nursing staff, 19 were nursing sisters, 51 staff $\vec{D}$ nurses, 23 student nurses and 7 auxiliary nurses. 
Table I shows the vaccination status of the various groups and highlights the considerable differences between medical and nursing staff as well as the large proportion of subjects who are unprotected.

Table II lists the reasons given for not receiving the vaccine. Again there were some striking differences between the two groups.

Four $(6 \%)$ of the nurses and $7(21 \%)$ of the doctors who had not been vaccinated had attempted to be immunized but had given up due to difficulty obtaining the vaccine. This trend towards medical staff experiencing difficulty with vaccination was also seen in the immunized group. The majority of the vaccine was prescribed by general practitioners $(92 \%$ amongst nurses and $61 \%$ amongst doctors) but amongst medical staff a significant amount $(24 \%)$ was prescribed by friends or colleagues. Amongst doctors, $46 \%$ considered that no one had ever advised them to be vaccinated compared with $35 \%$ of nurses. The commonest source of advice for both groups was professional colleagues (nurses $36 \%$, doctors $35 \%$ ) followed by occupational health $(27 \%$ for nurses but only $7 \%$ for doctors). General practitioners provided little advice to either group (nurses $5 \%$, doctors $1 \%$ ). Amongst the unvaccinated subjects, $93 \%$ of nurses and $60 \%$ of doctors indicated that they wished to be immunized.

Fifty seven per cent of nurses and $87 \%$ of doctors could recall one or more needlestick injuries with $5 \%$ of nurses and $19 \%$ of doctors reporting greater than 10 such events.

\section{Discussion}

It is generally accepted that hepatitis B vaccination is justified amongst health care workers ${ }^{7}$ and that failure to immunize places them at unnecessary risk. However, the available data suggest that hepatitis B vaccine uptake is generally poor which is confirmed in this study.

The overall rate of immunization in the hospital-

Table I Rate of uptake of hepatitis B vaccine

\begin{tabular}{lccccc}
\hline & $n$ & $\begin{array}{c}\text { Percentage vaccinated } \\
\text { and tested for } \\
\text { seroconversion }\end{array}$ & $\begin{array}{c}\text { Percentage vaccinated } \\
\text { but }\end{array}$ & not tested & $\begin{array}{c}\text { Partially } \\
\text { vaccinated } \\
\text { vaccinated }\end{array}$ \\
\hline Medical staff & & & & & \\
Consultant & 25 & 16.0 & 12.0 & 12.0 & 60.0 \\
Senior registrar & 14 & 14.3 & 14.3 & 28.6 & 42.9 \\
Registrar & 14 & 42.9 & 0 & 21.4 & 35.7 \\
Senior house officer & 28 & 42.9 & 25.0 & 14.3 & 17.9 \\
House officer & 19 & 36.8 & 31.6 & 21.0 & 10.5 \\
Total medical staff & 100 & 31.0 & 18.0 & 18.0 & 33.0 \\
Nursing staff & & & & 10.5 & 5.8 \\
Sister & 19 & 21.0 & 11.8 & 13.7 & 62.7 \\
Staff Nurse & 51 & 11.8 & 4.3 & 13.0 & 60.9 \\
Student Nurse & 23 & 21.7 & 0 & 14.3 & 71.4 \\
Auxiliary Nurse & 7 & 14.3 & 9.0 & 14.0 & 61.0 \\
Total Nursing Staff & 100 & 16.0 & & & 52.6 \\
\hline
\end{tabular}

Table II Reasons given for not receiving vaccine

\begin{tabular}{lcc}
\hline & $\begin{array}{c}\text { Percentage of } \\
\text { unvaccinated } \\
\text { medical staff }(n=33)\end{array}$ & $\begin{array}{c}\text { Percentage of } \\
\text { unvaccinated } \\
\text { nursing staff }(\boldsymbol{n}=61)\end{array}$ \\
\hline Apathy & 39.4 & 18.0 \\
$\begin{array}{l}\text { Difficulty obtaining } \\
\quad \text { vaccine }\end{array}$ & 24.2 & 13.1 \\
Fear of vaccine & 0 & 36.1 \\
Not advised & 9.1 & 27.9 \\
Not at risk & 12.1 & 9.8 \\
Previous hepatitis B & 6.1 & 0 \\
$\quad$ infection & 9.1 & 4.9 \\
Others & & \\
\hline
\end{tabular}


based medical staff in this study $(49 \%)$ is in accord with a recently published figure for general practitioners $(48 \%)^{6}$ but conceals a wide variation between house officers and consultants. The high rate of uptake amongst house officers is encouraging as this is a group at particularly high risk. ${ }^{8}$ However, as this study shows that many junior doctors experience needlestick injuries early in their careers it would seem preferable to immunize them as medical students before clinical exposure. The uptake amongst senior medical $(28 \%)$ and all nursing $(25 \%)$ staff was uniformly disappointing and is a problem that needs to be addressed. It is well known that not all individuals seroconvert following hepatitis B immunization. ${ }^{9}$ Approximately one third of subjects in this study had not had confirmation of immunity possibly giving some a false sense of security.

The policy of identifying particularly high risk groups for immunization, such as those working on dialysis units, is probably outdated. Junior nurses rotate through many specialties and may be asked to 'relieve' in high risk areas at times of staff shortages. Similarly, junior doctors cannot be

\section{References}

1. Welch, J., Webster, M., Tilzey, A. et al. Hepatitis B infections after gynaecological surgery. Lancet 1989, i: 205-207.

2. Polakoff, S. Acute viral hepatitis B: laboratory reports 1980-4. Br Med J 1986, 293: 37-38.

3. Zuckerman, A.J. Time for action on hepatitis B immunisation. $\mathrm{Br}$ Med J 1987, 294: 771.

4. Harward, M.P., Kaiser, D.L. \& Fedson, D.S. Acceptance of hepatitis B vaccine by medical and surgical residents. J Gen Intern Med 1988, 3: 150-155.

5. Editorial. Few physicians vaccinated against hepatitis B. Wisc Med J 1989, 88: 14.

6. Kinnersley, P. Attitudes of general practitioners towards their vaccination against hepatitis B. Br Med J 1990, 300: 238 .

7. Zuckerman, A.J. Who should be immunised against hepatitis B? Br Med J 1984, 289: 1243-1244. realistically classified into high or low risk groups. The strong potential for infection in all groups is also indicated by the remarkably high incidence of needlestick injury.

In view of the fact that the majority of unprotected individuals in this study indicated a desire to be immunized it is important to consider ways of ensuring a better uptake of the vaccine. Amongst nursing staff, lack of advice and fear of the vaccine were important factors and both should be amenable to better education campaigns. Apathy, a particularly prominent feature amongst medical staff, is a much more difficult problem to address. It seems reasonable to assume that the policy of passing responsibility for immunization to general practitioners must place an unnecessary hurdle in front of potential vaccinees. There is some evidence to suggest poor uptake even when the vaccine is made freely available ${ }^{10}$ although it is only recently that the safety of the vaccine has been generally appreciated.

A re-appraisal of attitudes towards the provision of hepatitis B vaccine is necessary but the cost implications would be considerable.

8. Snydman, D.R., Munoz, A., Werner, B.G. et al. A mult variate analysis of risk factors for hepatitis B virus infection among hospital employees screened for vaccination. $A m$ Epidemiol 1984, 120: 684-693.

9. Laukamm-Josten, U., von Laer, G., Feldmeier, H. et al. Active immunisation against hepatitis B: immunogenicity of a recombinant DNA vaccine in females, heterosexual and homosexual males. Postgrad Med J 1987, 63 (Suppl. 2): $143-146$.

10. Scapa, E., Karpuch, J., Waron, M. et al. Attitude of hospital personnel toward hepatitis B vaccination. Am J Gastroentero 1989, 84: 400-402. 